\title{
Results of wrist extension reconstruction in C5-8 brachial plexus palsy by transferring the pronator quadratus motor branch to the extensor carpi radialis brevis muscle
}

\author{
Jayme Augusto Bertelli, MD, PhD, ${ }^{1,2}$ Marcos Flávio Ghizoni, MD, MSc, ${ }^{1}$ and \\ Cristiano Paulo Tacca, MD² \\ 'Department of Neurosurgery, Southern University of Santa Catarina (UNISUL), Tubarão; and 2Department of Orthopedic \\ Surgery, Governador Celso Ramos Hospital, Florianópolis, Santa Catarina, Brazil
}

\begin{abstract}
OBJECTIVE The objective of this study was to report the results of pronator quadratus (PQ) motor branch transfers to the extensor carpi radialis brevis (ECRB) motor branch to reconstruct wrist extension in C5-8 root lesions of the brachial plexus.

METHODS Twenty-eight patients, averaging 24 years of age, with C5-8 root injuries underwent operations an average of 7 months after their accident. In 19 patients, wrist extension was impossible at baseline, whereas in 9 patients wrist extension was managed by activating thumb and wrist extensors. When these 9 patients grasped an object, their wrist dropped and grasp strength was lost. Wrist extension was reconstructed by transferring the PQ motor to the ECRB motor branch. After surgery, patients were followed for at least 12 months, with final follow-up an average of 22 months after surgery.
\end{abstract}

RESULTS Successful reinnervation of the ECRB was demonstrated in 27 of the 28 patients. In 25 of the patients, wrist extension scored M4, and in 2 it scored M3.

CONCLUSIONS In C5-8 root injuries, wrist extension can be predictably reconstructed by transferring the $\mathrm{PQ}$ motor branch to reinnervate the ECRB.

http://thejns.org/doi/abs/10.3171/2015.3.JNS142428

KEY WORDS brachial plexus; wrist extension; nerve grafting; nerve transfer; neurotization; peripheral nerve

$\mathrm{T}$ Raditionally, it has been accepted that if the C5-7 roots are injured, there will be palsy of shoulder abduction/external rotation, elbow flexion/extension, and thumb and finger extension. ${ }^{1}$ Although this belief is widely held, it is inconsistent with well-accepted myotome charts for the upper limb. ${ }^{9}$ For instance, it is accepted that thumb and finger extensors are innervated by the C-7 and C-8 roots; consequently, preservation of thumb and finger span should be observed in C5-7 root injuries. Bertelli and Ghizoni ${ }^{4}$ have demonstrated that C5-7 root injuries do not cause palsies of elbow, wrist, or finger extension. In such cases, radial wrist extensors are paralyzed, but strong wrist extension is preserved because the extensor carpi ulnaris remains functional. Bertelli and Ghizoni ${ }^{3}$ have also demonstrated that what was clinically conceived to be a C5-7 root palsy may in fact be a C5-8 root injury. Fattah et al. reached the same conclusions in children. ${ }^{12}$ In a few patients with C5-8 root palsies, thumb and finger extension is preserved because the $\mathrm{T}-1$ root participates in the innervation of thumb and finger extensors. ${ }^{3}$ In these patients, if thumb and finger extensors are strong enough, wrist extension can be accomplished using these muscles. ${ }^{3}$ However, concomitant with the patient flexing his or her fingers to grasp an object, the wrist drops because there is no active wrist extensor to maintain the wrist in extension. When the wrist is flexed, grasp power is essentially lost. ${ }^{15}$ In C5-8 root injuries, such patients, as well as those patients with clear palsies of wrist extension, need reconstruction of wrist extensors.

Very few options are available for wrist extensor reconstruction in such extended upper-type palsies of the upper limb, because only a limited number of donor nerves and tendons are available. Common donors for tendon transfers to correct a dropped wrist include the pronator teres

ABBREVIATIONS AIN = anterior interosseous nerve; ECRB = extensor carpi radialis brevis; FCU = flexor carpi ulnaris; FDP = flexor digitorum profundus; FDS = flexor digitorum superficialis; $\mathrm{FPL}$ = flexor pollicis longus; $\mathrm{MRC}=$ Medical Research Council; $\mathrm{PQ}=$ pronator quadratus; $\mathrm{PT}$ = pronator teres.

SUBMITTED October 25, 2014. ACCEPTED March 30, 2015.

INCLUDE WHEN CITING Published online October 2, 2015; DOI: 10.3171/2015.3.JNS142428. 
(PT), which is paralyzed in C5-8 root injuries, and tendons of the flexor digitorum superficialis (FDS), which are partially paralyzed. ${ }^{13} \mathrm{Ukrit}$ et al. ${ }^{19}$ proposed transferring a motor branch nerve of the FDS to reinnervate the extensor carpi radialis brevis (ECRB). More recently, Bertelli et al. ${ }^{8}$ have proposed transfers of the pronator quadratus (PQ) motor branch-which is the terminal division of the anterior interosseous nerve (AIN) and receives a substantial contribution from the T-1 root-to the ECRB motor branch. They reported the results for wrist extension reconstruction in 4 patients.

In the present paper, we describe the results of transferring the PQ motor branch in a series of 28 consecutive patients with C5-8 brachial plexus root injuries, formerly known as C5-7 root palsies. We analyzed both the recovery of elbow flexion and wrist pronation-supination. We advance the "Oberlin effect" concept, which is defined as elbow flexion coupled with wrist flexion, i.e., every time the patient flexes his or her elbow, his or her wrist concomitantly flexes, leading to poor hand posture for grasping. This results from the absence of wrist extensors and from the Oberlin procedure itself, because it transfers ulnar nerve motor fibers normally directed toward the flexor carpi ulnaris (FCU) to the biceps motor branch for elbow flexion reconstruction. ${ }^{16}$ In our series, we demonstrated that the Oberlin effect can be eliminated by reinnervating the ECRB via transfer of the PQ motor branch.

\section{Methods \\ Patient Population}

From December 2011 to April 2014, 40 patients with C5-8 root injuries of the brachial plexus underwent surgical reconstruction. Among these 40 patients, 27 males and 1 female were followed for at least 12 months and are the subject of the present report. The patients' average age was 24 years (range 16-48 years). The mean interval between the accident and surgery was 6.7 months (range 2-18 months). Before surgery, every patient underwent cervical helical CT myelography following intradural contrast injection to evaluate the roots of the brachial plexus intradurally, and to detect if roots were avulsed or preserved. All patients exhibited palsy of shoulder abduction, external rotation, and elbow flexion. Elbow extension was preserved, scoring M4 on the British Medical Research Council (MRC) scale in 3 patients in whom a triceps motor branch was used to reinnervate the axillary nerve.

All patients also had palsy of their wrist extensors. However, in 9 of the 28 patients, wrist extension was possible because the thumb and finger extensors remained functional. When the fingers were placed in the intrinsic minus position, as suggested previously, ${ }^{3}$ wrist extension from full flexion was no longer possible, confirming that wrist extension was mediated by finger and thumb extensors. In all of these patients, when they grabbed any object, their wrist dropped. In all of the patients, the PT was paralyzed but pronation was preserved and scored M4. In all, thumb interphalangeal joint and index distal interphalangeal joint flexion scored M4, denoting preservation of AIN function. All patients subsequently underwent operations and the brachial plexus was explored for repair.

\section{Operative Procedures}

When available, C-5 and C-6 roots were grafted to the upper trunk $(n=3)$. If only $C-5$ was available, it was grafted to the anterior division of the upper trunk $(n=2)$, posterior division of the upper trunk $(n=6)$, or the middle trunk $(n=2)$. In 1 patient the upper trunk was grafted to the axillary nerve. The accessory nerve was transferred to the suprascapular nerve. Elbow flexion was reconstructed by transferring motor fascicles of the ulnar nerve preferentially related to finger flexion and intrinsic hand muscle function to the biceps motor branch, as reported previously. ${ }^{5}$ If elbow extension was reconstructed by transferring 1 motor branch of the FCU to a triceps branch $(n=5)$, elbow flexion was reconstructed by transferring motor fascicles of the median nerve, rather than the ulnar nerve, to the biceps motor branch.

In all patients, we transferred the motor branch of the PQ muscle to the ECRB, as previously described. ${ }^{8}$ The AIN was dissected via a skin incision centered over the wrist, extending from $8 \mathrm{~cm}$ proximal to the wrist volar crease to the antecubital fossa (Figs. 1 and 2). After skin incision, the median nerve was located and its accessory branch to the FDS of the index finger identified. Deep dissection was then performed between the flexor digitorum profundus (FDP) and flexor pollicis longus (FPL) to identify the interosseous membrane and locate the AIN. After the AIN was located, it was followed proximally, and for this step the FDS was detached from the radius. Dissection stopped at the distal margin of the PT. At this level, care was taken not to damage the FPL main motor branch. To confirm functionality, the AIN nerve was electrically stimulated and contractions of the PQ were observed. Nerves were stimulated using an insulated 21-gauge needle (Contiplex D; B. Braun Melsungen AG) connected to a nerve stimulator (Stimuplex HNS 11; B. Braun Melsungen AG). Stimulus intensity ranged from 1 to $5 \mathrm{~mA}$. Next, the superficial radial nerve was located at the proximal part of the incision, and deep and parallel to it we identified the motor branch of the ECRB, as previously described. ${ }^{4}$ The branch of the ECRB was sectioned at its origin and flipped distally. The AIN was sectioned $3-4 \mathrm{~cm}$ proximally to the PQ proximal border and tunneled under the flexor carpi radialis (FCR), PT, and radial artery toward the ECRB motor branch. Any redundant length of the recipient nerve was trimmed out and nerve coaptation performed as close as possible to the motor entry point, before the first division of the motor branch to the ECRB. The distal stump of the AIN was connected to the distal median nerve branch to the FDS of the index finger, previously dissected in the distal forearm. Nerve coaptation was performed using 9-0 monofilament sutures (Figs. 1 and 2). In the 3 patients with fixed forearm rotation the distal stump of the AIN was not reinnervated. A patient data summary and details of the surgical procedure are listed in Table 1.

\section{Postoperative Control and Follow-up}

After surgery, patients used an arm sling for 1 week. Patients were followed up for at least 12 months, up to an average follow-up duration of $22 \pm 7$ months postoperatively, at which point a thorough final evaluation was performed. During each medical visit, patients were asked to 


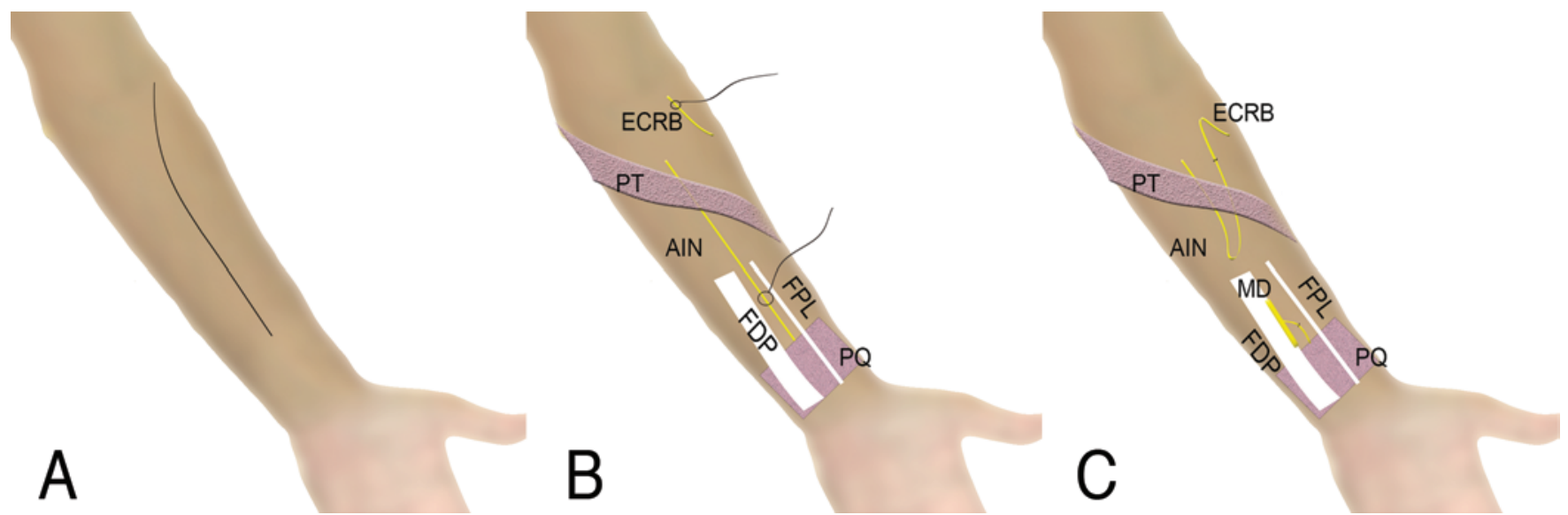

FIG. 1. Schematic representation of the surgical incision (A) and surgical technique (B and C) used to transfer the AIN to the ECRB motor branch. In the wrist, the AIN lies between the tendons of the FPL and FDP (B). After dividing the AIN 3-4 cm proximal to the $P Q$, the AIN is tunneled under the FCR, the PT, and the radial artery toward the ECRB motor branch. The distal stump of the AIN is sutured to a branch of the median nerve (MD) to the FDS of the index finger (C). Figure is available in color online only.

pronate against resistance and, when contractions of the ECRB were identified, patients were taught that pronation produced wrist extension. They were instructed to practice motion at home, at least 4 times a day. Initially, they practiced with the elbow extended alongside their body to eliminate gravity. They received no special physiotherapy training.

Reinnervation of the ECRB was confirmed by direct tendon palpation of its insertion into the base of the second metacarpal bone. During active wrist extension and finger flexion with the wrist extended, tendon motion of the ECRB was resisted by having the examiner's fingertip pressing against it, as reported elsewhere. ${ }^{2}$ Wrist extension strength was manually assessed using the British MRC strength scale. ${ }^{20}$ Patients were asked to flex their elbow, and wrist position was noted. If wrist flexion occurred concomitantly with elbow flexion, the occurrence of the Oberlin effect was ascertained.

Elbow flexion/extension strength was also assessed using the BRMC scale. ${ }^{20}$ With the elbow flexed to $90^{\circ}$ of flexion and supported on a table, active and passive range of motion for pronation-supination was visually estimated. Pronation range was measured from full supination, and vice versa. We considered $160^{\circ}$ as the normal range of pronation-supination. ${ }^{14}$ Pre- and postoperative evaluations were performed by the surgeon (J.A.B.) who operated on the patients.

\section{Results}

In 25 of the 28 patients, wrist extension scored M4; while in 2, it scored M3, and in 1, M2. In those patients scoring M4 or M3, full range of wrist extension was ob-

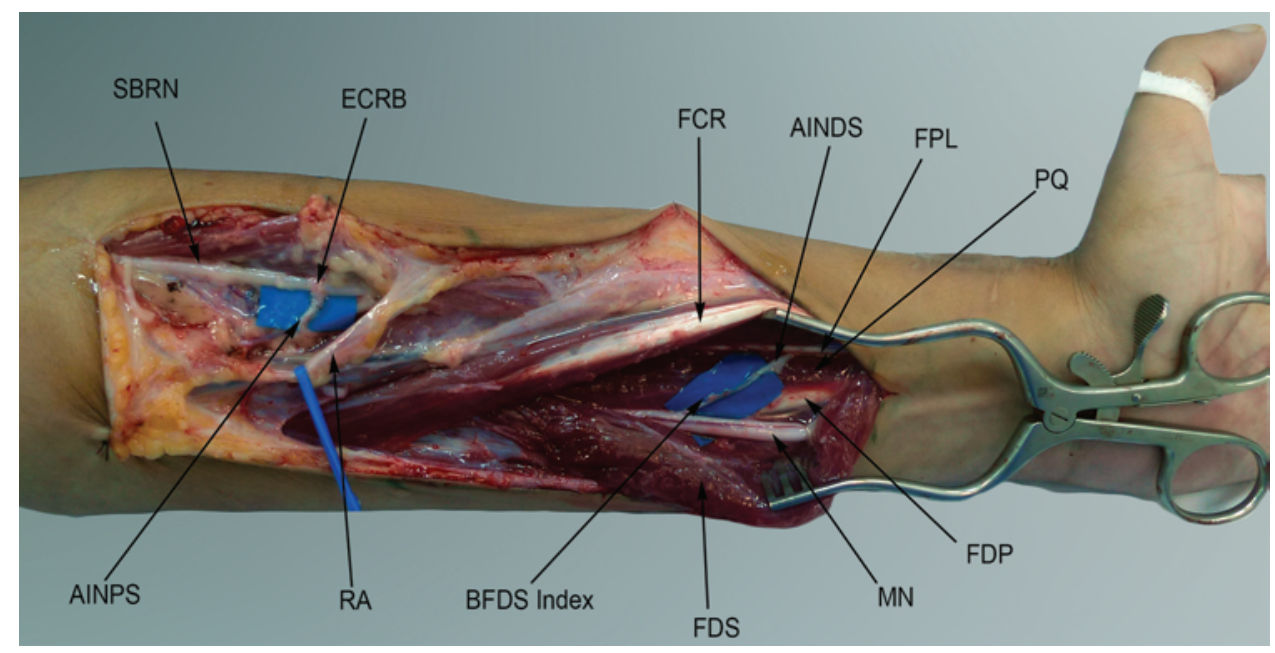

FIG. 2. Intraoperative view of the transfer of the AIN to the ECRB motor branch. AINPS = AIN proximal stump, which was connected to the ECRB motor branch to reconstruct wrist extension; BFDS Index = secondary branch of the median nerve (MN) to the FDS of the index, which was connected to the AIN distal stump (AINDS) to restore pronation; RA = radial artery; SBRN = superficial branch of the radial nerve. Figure is available in color online only. 


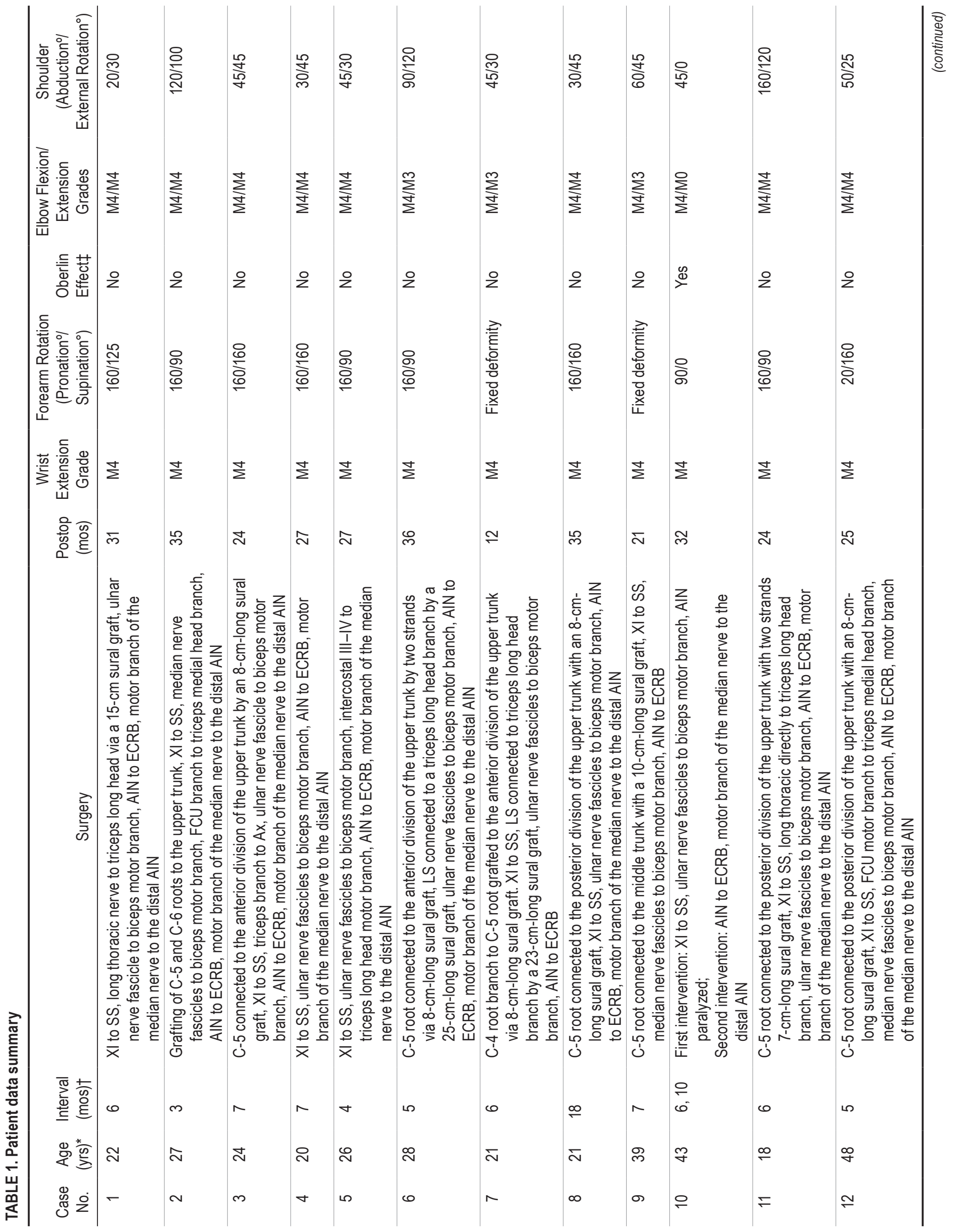




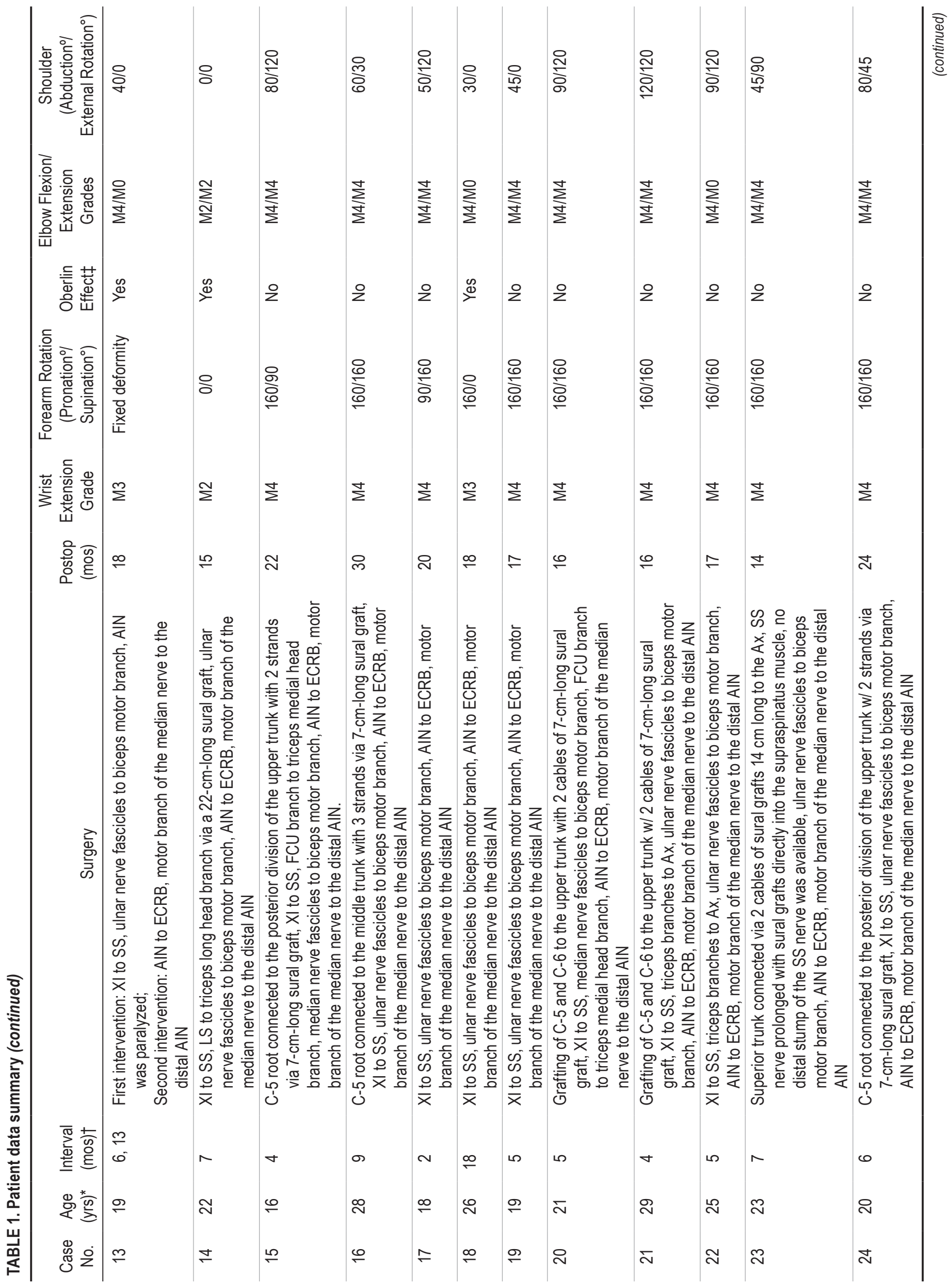




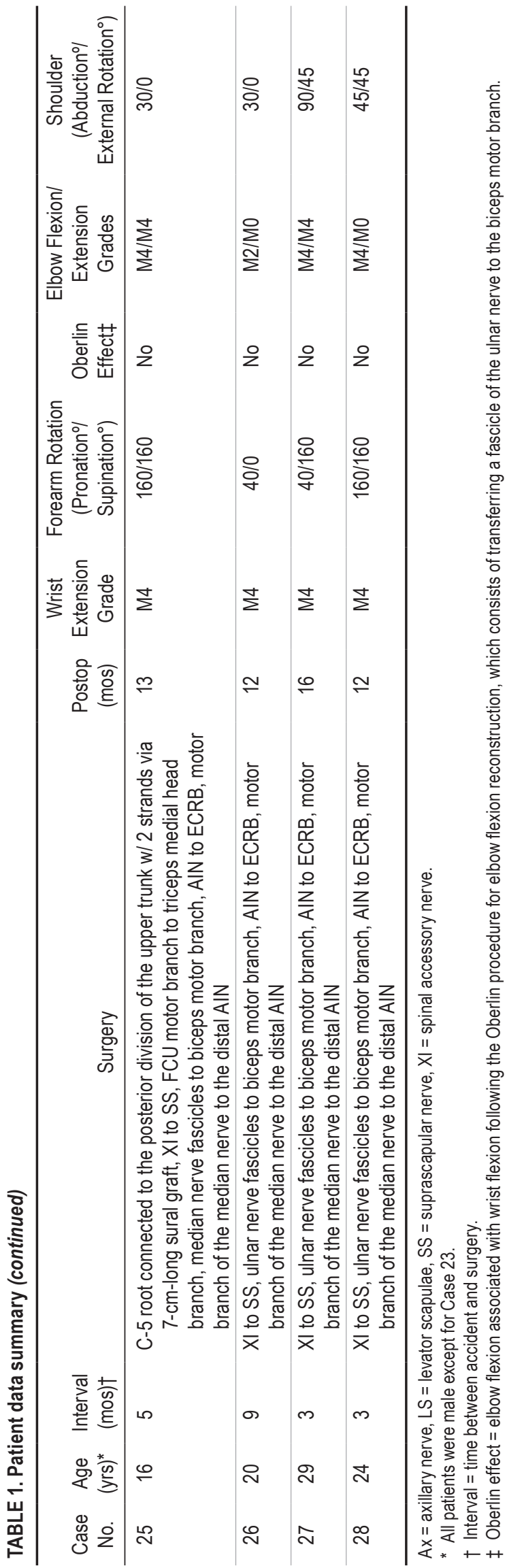

served. Postoperatively, we did not observe any functional downgrading of the FPL or FDP of the index and middle fingers because of manipulation and sectioning of branches of the AIN to these muscles in the distal forearm.

Twenty-six patients recovered elbow flexion represented by an M4 score, and 2 with a score of M2. Initially, when flexing the elbow, all patients also flexed their wrist (i.e., the Oberlin effect). When wrist extension scored M4, the elbow could be flexed without concomitant flexion of the wrist.

Among the 26 patients with elbow flexion recovery, 3 had fixed forearm rotation. In all of them the forearm was fixed around $30^{\circ}$ of pronation; in none of them was the forearm fixed in full supination. Hence, of the 23 patients with full passive range of forearm rotation and biceps M4 level of strength recovery, complete range of active supination was observed in 15 patients, while supination paralysis was observed in 3; 5 patients demonstrated incomplete range of supination, with an average range of $95^{\circ}$. Pronation was complete in 19 of the 25 patients with supple forearm rotation, and incomplete in 5, who exhibited an average range of $55^{\circ}$. Following surgery, the range of pronation was decreased in 6 patients, but only 1 of them complained about it. Global results for shoulder, elbow flexion/ extension, and wrist extension are summarized in Table 1.

\section{Discussion}

For closed C5-8 root injuries of the brachial plexus, we indicate surgery if elbow flexion fails to recover by the third month. In partial injuries, surgery can be postponed if some function has already been restored, but ideal conditions for nerve transfers have not yet been identified. For instance, in some patients with a C5-8 root injury, if by the third month finger flexion is present but still short of an M4 level of strength, surgery may be postponed, with M4 grasping strength the ideal condition for transferring ulnar nerve fascicles to the biceps motor branch. ${ }^{16}$ Under these circumstances, we prefer to postpone surgery for up to 6 months, allowing for spontaneous recovery from any axonotmetic injury, thereby trying to eliminate the possibility of transferring a poor donor nerve. Because distal nerve transfers are performed close to motor endplates, the time interval between injury and surgery is less critical relative to nerve grafts. In another situation, when initially examined patients with a C5-8 root injury may be unable to pronate their forearm, if patients rapidly experience the initiation of recovery of thumb and index distal phalanx flexion, recovery of PQ function can be anticipated because these functions are all mediated by the AIN. Surgery can be postponed to allow for adequate reinnervation of the PQ muscle to an M4 level of strength, ensuring a good donor nerve for transfer. Patients with C5-8 root injuries and CT myelographs confirming the lesion, in particular C7-8 root avulsion and T-1 preservation with adequate donors for transfer, can be operated upon without delay. This occurred in 7 of our patients who underwent operations within 4 months of their injury. Nine of our patients were not operated on before 6 months, because of late referral, socioeconomic issues, or late recovery of $\mathrm{PQ}$ function. Those patients with late recovery of $\mathrm{PQ}$ function 
ultimately underwent 2 surgical procedures, the first surgery primarily to reconstruct shoulder and elbow flexion.

In our series of 28 patients, transfer of the PQ motor branch to the ECRB motor branch resulted in a high rate of successful wrist extension reconstruction, with only 1 patient achieving no measurable benefit. Two patients scored M3 at 12 months, and it is quite possible that with longer follow-up they ultimately would have achieved M4level strength. M4 strength or better is needed for fully functional wrist extension, which allows patients to grab objects without flexing their wrist. Patients with active wrist extension driven by thumb and finger extensors benefited from reinnervation of the ECRB, because after surgery they could grasp strongly without flexing their wrist.

After harvesting the AIN in the distal forearm, none of the patients demonstrated paralysis or loss of strength of the FPL and FDP, because the main motor branches of these muscles originate from the AIN proximally to the PT. ${ }^{7,8}$ Preoperatively, direct PQ palpation to determine function preservation is not possible. However, because of palsy of the PT in C5-8 root injury, any active pronation should originate from the contraction of the PQ. In addition, preservation of FPL and FDP of the index finger suggests preservation of the AIN and further confirms the availability of the PQ motor branch as a healthy donor nerve for transfer.

In those patients with partial upper-type lesions of the brachial plexus, the most rewarding method of elbow flexion reconstruction is transferring ulnar fascicles to the FCU to the biceps motor branch. ${ }^{21}$ However, in C5-8 root injuries, when wrist extensors are paralyzed this method has one tremendous drawback: when patients flex their elbow, their wrist flexes too, because elbow flexion is linked to activation of the FCU, a prime wrist flexor. This coactivation leads to awkward positioning of the hand during elbow flexion, significantly compromising routine hand use. In these patients, we prefer to avoid harvesting ulnar nerve fascicles to the FCU. We prefer to harvest nerve fascicles of the ulnar nerve to the FDP or to intrinsic muscles of the hand. There is no downgrading of hand function when this strategy is employed. ${ }^{5}$ More important, however, is to reconstruct wrist extension, as demonstrated herein, to eliminate what we propose calling the "Oberlin effect" (i.e., elbow flexion coupled with awkward wrist flexion following an Oberlin nerve transfer ${ }^{16}$ ).

Pre- and postoperative evaluations were conducted by the surgeon who operated on the patients. This could have been a source of bias. However, we believe that if bias existed it would be minimal, because the major focus of the study was on single-function reconstruction, specifically wrist extension. Postoperatively, only 3 scores were possible: failure of wrist extension recovery, wrist extension recovery against gravity, and wrist extension recovery against resistance. We have made our pre- and postoperative evaluations based on the range of motion and strength recovery. We have not used any scale of functional disability that could have shed light on the importance of wrist extension and pronation-supination recovery in daily activities.

Alternatives to PQ motor branch transfer for wrist extension reconstruction are very limited and include the transfer of either motor branches ${ }^{6,19}$ or tendons of the FDS..$^{13}$ However, in our hands, transferring fascicles of the FDS to the ECRB motor branch yields poor results in patients with $\mathrm{C} 5-8$ root injuries. ${ }^{6}$ Tendon transfers using the FDS of the third and fourth fingers to the ECRB tendons, through the interosseous membrane, is our preferred alternative, but this strategy may decrease hand strength.

Although we restored full elbow flexion in $92 \%$ of our cases, the same could not be said about supination. Among the 23 patients with biceps reinnervation and full range of passive pronation-supination, full active supination was restored in only $15(65 \%)$ of them. Considering the reinnervated biceps the sole supinator in C5-8 root injury, we believe that the lack of supination is associated with less than ideal biceps strength recovery, even for those patients scoring M4, because M4 may be associated with only $10 \%$ of normal strength. ${ }^{11}$ Of importance is that, in C5-8 root injuries, the ulnar nerve (i.e., the donor nerve) is partially injured as it is formed by the $\mathrm{C} 8-\mathrm{T} 1$ roots, with a predominant contribution coming from C-8. ${ }^{10}$ This also accounts for differences in elbow flexion recovery between C5-6 root injuries and more extended upper-type palsies using nerve transfers from the ulnar nerve to reinnervate the biceps. ${ }^{16}$

In $\mathrm{C} 5-8$ root lesions, the PT is paralyzed and active pronation relies upon the PQ. ${ }^{8}$ In this situation, harvesting the PQ motor branch will hamper pronation. We have successfully reconstructed pronation by transferring a distal motor branch of the FDS to the index finger to the distal branch of the AIN in 19 patients. Partial recovery was observed in an additional 5 patients. It is possible that not only our nerve transfer, but also the pull of finger flexors contributed to active pronation. ${ }^{8}$ In 2 patients, pronation was not restored. Six patients had their preoperative status downgraded by surgery. However, only 1 complained about losing pronation. We believe that the recovery of wrist extension outweighs any deficits in pronation, however, provided also that supination cannot be predictably restored by biceps reinnervation in these patients. Pronation is useless in the wrist drop, and if supination is not reconstructed. If we had added the transfer of a redundant branch of the FDS to the PT motor branch as suggested previously ${ }_{18}^{18}$ we probably would have improved our results in pronation reconstruction. However, we chose not to perform this step because we were afraid of losing FDS strength following the already existing partial denervation as a consequence of the extended root injury, which ultimately would compromise hand grasping and late tendon transfer in cases of failure of wrist extension recovery.

Patients with thumb, finger, and wrist extension palsies cope reasonably well with finger extension paralysis, but poorly with wrist drop..$^{17}$ In C5-8 palsies, surgeons should endeavor to reconstruct wrist extension. In our series, transfer of the PQ motor branch to the ECRB was highly successful, yielding M4-level strength in $90 \%$ of our patients. This is a clearly superior result to when the FDS is used as the donor for nerve transfer, given that in the reported series, M4-level strength was never achieved and patients had poor control of their restored wrist extension. ${ }^{6}$ Provided that the FCU is functional, thumb and finger extension can be addressed later on by transferring the FCU 
tendon to the extensor digitorum communis and extensor pollicis longus.

\section{Conclusions}

In C5-8 root injuries, wrist extension can be predictably reconstructed by transferring the PQ motor branch to reinnervate the $\mathrm{ECRB}$.

\section{References}

1. Barnes R: Traction injuries of the brachial plexus in adults. $\mathbf{J}$ Bone Joint Surg Br 31B:10-16, 1949

2. Bertelli JA: Tendon palpation during agonist contraction and antagonist co-contraction to assess wrist flexors and extensors muscle function. Chir Main 34:79-85, 2015

3. Bertelli JA, Ghizoni MF: C5-8 brachial plexus root injury: the "T-1 hand". J Neurosurg 116:409-413, 2012

4. Bertelli JA, Ghizoni MF: Clinical findings in C5-C6 and C5C7 root palsies with brachial plexus traction lesions. J Hand Surg Eur Vol 38:237-241, 2013

5. Bertelli JA, Ghizoni MF: Nerve root grafting and distal nerve transfers for C5-C6 brachial plexus injuries. J Hand Surg Am 35:769-775, 2010

6. Bertelli JA, Ghizoni MF: Transfer of a flexor digitorum superficialis motor branch for wrist extension reconstruction in C5-C8 root injuries of the brachial plexus: a case series. Microsurgery 33:39-42, 2013

7. Bertelli JA, Mendes Lehm VL, Tacca CP, Winkelmann Duarte EC, Ghizoni MF, Duarte H: Transfer of the distal terminal motor branch of the extensor carpi radialis brevis to the nerve of the flexor pollicis longus: an anatomic study and clinical application in a tetraplegic patient. Neurosurgery 70:1011-1016, 2012

8. Bertelli JA, Tacca CP, Winkelmann Duarte EC, Ghizoni MF, Duarte $\mathrm{H}$ : Transfer of the pronator quadratus motor branch for wrist extension reconstruction in brachial plexus palsy. Plast Reconstr Surg 130:1269-1278, 2012

9. Brendler SJ: The human cervical myotomes: functional anatomy studied at operation. J Neurosurg 28:105-111, 1968

10. Chiba T, Konoeda F, Higashihara M, Kamiya H, Oishi C, Hatanaka Y, et al: C8 and T1 innervation of forearm muscles. Clin Neurophysiol 126:837-842, 2015

11. Dvir Z: Grade 4 in manual muscle testing: the problem with submaximal strength assessment. Clin Rehabil 11:36-41, 1997

12. Fattah A, Curtis CG, Agur AMR, Clarke HM: Functional contribution of $\mathrm{T} 1$ to the brachial plexus in infants. J Hand Surg Eur Vol 37:237-243, 2012

13. Krishnan KG, Schackert G: An analysis of results after selec- tive tendon transfers through the interosseous membrane to provide selective finger and thumb extension in chronic irreparable radial nerve lesions. J Hand Surg Am 33:223-231, 2008

14. Hausman MR, Lang P: Examination of the elbow: current concepts. J Hand Surg Am 39:2534-2541, 2014

15. Li ZM: The influence of wrist position on individual finger forces during forceful grip. J Hand Surg Am 27:886-896, 2002

16. Loy S, Bhatia A, Asfazadourian H, Oberlin C: [Ulnar nerve fascicle transfer onto to the biceps muscle nerve in C5-C6 or C5-C6-C7 avulsions of the brachial plexus. Eighteen cases.] Ann Chir Main Memb Super 16:275-284, 1997 (Fr)

17. Smith RJ: Tendon Transfers of the Hand and Forearm. Boston: Little, Brown \& Co, 1987

18. Tung TH, Mackinnon SE: Flexor digitorum superficialis nerve transfer to restore pronation: two case reports and anatomic study. J Hand Surg Am 26:1065-1072, 2001

19. Ukrit A, Leechavengvongs S, Malungpaishrope K, Uerpairojkit C, Chongthammakun S, Witoonchart K: Nerve transfer for wrist extension using nerve to flexor digitorum superficialis in cervical 5, 6, and 7 root avulsions: anatomic study and report of two cases. J Hand Surg Am 34:1659-1666, 2009

20. Vanhoutte EK, Faber CG, van Nes SI, Jacobs BC, van Doorn PA, van Koningsveld R, et al: Modifying the Medical Research Council grading system through Rasch analyses. Brain 135:1639-1649, 2012

21. Yang LJ, Chang KW, Chung KC: A systematic review of nerve transfer and nerve repair for the treatment of adult upper brachial plexus injury. Neurosurgery 71:417-429, 2012

\section{Disclosure}

The authors report no conflict of interest concerning the materials or methods used in this study or the findings specified in this paper.

\section{Author Contributions}

Conception and design: Bertelli. Acquisition of data: all authors. Analysis and interpretation of data: Ghizoni. Drafting the article: Bertelli. Critically revising the article: Bertelli, Ghizoni. Reviewed submitted version of manuscript: Bertelli. Approved the final version of the manuscript on behalf of all authors: Bertelli.

\section{Correspondence}

Jayme A. Bertelli, Department of Neurosurgery, Southern University of Santa Catarina [UNISUL], Rua Newton Ramos 70, apto 901, Florianópolis-SC, 88015395, Brazil. email: drbertelli@ gmail.com. 